\title{
HAUSDORFF SUMMABILITY METHODS, ADDENDUM
}

\author{
BY \\ B. E. RHOADES(1)
}

It is the purpose of this paper to answer the questions left unanswered in $\$ 4$ of [3]. For easy references, the statements to be proved are listed below. The reader is referred to [3] for the terminology used in this paper.

THEOREM 1. $H \sim \alpha_{2}(t, r)$ n.t.s. $H \sim \alpha_{3}(t, r)$.

THEOREM 2. For $b-1>-k / \log r, H \sim \alpha_{1}(t, k, b)$ n.t.s. $H \sim \alpha_{3}(t, r)$.

TheOREM 3. For $k>4, H \sim \alpha_{1}(t, k+1, c)$ n.t.s. $H \sim \alpha_{1}(t, k, c)$.

A generating sequence for a Hausdorff matrix is totally monotone if and only if the corresponding mass function is nonnegative and nondecreasing over the closed unit interval. The technique used in proving each of the above theorems is to show that the corresponding mass function is decreasing at at least one point in the unit interval.

Proof of Theorem 1. If we let $\mu(t)=\alpha_{2}(t, r) / \alpha_{3}(t, r)$, then $\mu(t)$ can be written as the product of $\lambda_{1}(t)$ and $\lambda_{2}(t)$, where

$$
\lambda_{1}(t)=\frac{\left(1-r^{t}\right)(t+1)}{t \log (1 / r)}, \lambda_{2}(t)=\frac{1-r}{1-r^{t+1}} .
$$

Let $\theta_{1}(u)$ and $\theta_{2}(u)$ denote the corresponding mass functions. Then, from $[2$, p. 781],

$$
\theta_{1}(u)= \begin{cases}0, & 0 \leqq u<r, \\ (1 / \log r) & -(\log u / \log r)+1, r \leqq u<1, \\ 1, & u=1,\end{cases}
$$

and

$$
\theta_{2}(u)=\left\{\begin{array}{l}
1, \quad u=1, \\
r^{k}, r^{k}<u<r^{k-1}, \quad k=1,2,3, \cdots .
\end{array}\right.
$$

If $\theta(u)$ denotes the mass function corresponding to $\mu(t)$, then, using the composition formula

Presented to the the Society, August 29, 1961, under the title Total relative strength of $a$ Hausdorff method; received by the editors January 30, 1962.

(1) This work was performed under the auspices of the U. S. Atomic Energy Commission. 


$$
\theta(u)=\theta_{2}(u)+\int_{u}^{1} \theta_{1}(u / v) d \theta_{2}(v)
$$

(see, e.g. $[1$, p. 196]) we obtain

$$
\theta(u)=\left\{\begin{array}{l}
\frac{-r^{k-1}}{\log r}[(1-r) \log u+(r(k-1)-k) \log r-(1-r)], \\
\quad r^{k}<u<r^{k-1}, \quad k=2,3,4, \cdots, \\
1+\frac{(1-r)(1-\log u)}{\log r}, r<u<1, \\
1, u=1 .
\end{array}\right.
$$

For $k=2,3,4, \cdots$,

$$
\theta\left(r^{k}+0\right)-\theta\left(r^{k}-0\right)=r^{k-1}(1-r)^{2} / \log r<0 .
$$

Therefore $\theta(u)$ is not a totally regular mass function.

Proof of Theorem 2. Now let $\mu(t)=\alpha_{1}(t, k, b) / \alpha_{3}(t, r)$. Then $\mu(t)$ can be written as the product of $\lambda_{1}(t)$ and $\lambda_{2}(t)$, where $\lambda_{1}(t)=\alpha_{1}(t, k, b) \cdot(t+1)$, and $\lambda_{2}(t)$ is as in Theorem 1 .

Writing $t+1$ as $b((t+b) / b)+1-b$, we may write $\lambda_{1}(t)$ in the form

$$
\lambda_{1}(t)=\frac{b}{k}+\frac{1}{k} \sum_{r=1}^{k-1}\left(\frac{b}{t+b}\right)^{r}+\left(\frac{b}{t+b}\right)^{k}\left(\frac{1-b}{k}\right) .
$$

If $\theta_{1}(t)$ denotes the mass function corresponding to $\lambda_{1}(t)$, then

$$
\theta_{1}(t)=\left\{\begin{aligned}
\frac{1}{k} & \sum_{r=1}^{k-1} \frac{b^{r}}{\Gamma(r)} \int_{0}^{u} t^{b-1}\left\{\log \frac{1}{t}\right\}^{r-1} d t \\
& \left.+(1-b) \frac{b^{k}}{\Gamma(k)} \int_{0}^{u} t^{b-1}\left\{\log \frac{1}{t}\right\}^{k-1} d t\right], 0 \leqq u<1 \\
1, u=1 &
\end{aligned}\right.
$$

Again using formula (1),

$$
\theta(t)=\left\{\begin{array}{c}
1, u=1, \quad r^{m}+(1-r) \sum_{j=0}^{m-1} \frac{r^{j}}{k}\left[\sum_{s=1}^{k-1} \frac{b^{s}}{\Gamma(s)} \int_{0}^{u r^{-j}} t^{b-1}\left\{\log \frac{1}{t}\right\}^{s-1} d t\right. \\
\left.+\frac{(1-b) b^{k}}{\Gamma(k)} \int_{0}^{u r^{-j}} t^{b-1}\left\{\log \frac{1}{t}\right\}^{k-1} d t\right], r^{m}<u<r^{m-1}, \\
m=1,2,3, \cdots .
\end{array}\right.
$$

For $r^{m}<u<r^{m-1}$,

$$
\frac{d \theta(u)}{d u}=\frac{(1-r)}{k} \sum_{j=0}^{m-1}\left(u r^{-j}\right)^{b-1} f(k, j)
$$


where

$$
f(k, j)=\sum_{s=1}^{k-1} \frac{b^{s}}{\Gamma(s)}\left\{\log \left(r^{j} / u\right)\right\}^{s-1}+\frac{(1-b) b^{k}}{\Gamma(k)}\left\{\log \left(r^{j} / u\right)\right\}^{k-1},
$$

(4) $f(k+1, j)-f(k, j)=\frac{b^{k+1}\left\{\log \left(r^{j} / u\right)\right\}^{k-1}}{k \Gamma(k)}\left[k+(1-b) \log \left(r^{j} / u\right)\right]$.

Case I. $k=2$. From (3), $f(2, j)<0$ whenever $1+(1-b) b \log \left(r^{j} / u\right)<0$; i.e., $u<a_{j}=r^{j} \exp (-1 / b(b-1))$. If we consider those values of $u$ in the interval $r<u<1$, then, from (2), $j=0$. We now determine a value $b_{0}$ such that we will have $u>r$ and $u<a_{0}<1$. Note that, since $b>1, r<a_{0}$ if and only if $b>b_{0}$, where $b_{0}=\left[-1+(1-(4 / \log r))^{1 / 2}\right] / 2$. Thus, for $r<u<a_{0}, f(2,0)<0$.

Case II. For $k>2$, again consider $u$ in the interval $r<u<1$; i.e., $j=$ 0 . Then (4) shows that $f(k, 0)$ is a decreasing function of $k$, provided $k-$ $(1-b) \log u<0$; i.e., $b>b_{1}=1-(k / \log r)$. It is easy to show that $b_{1}>b_{0}$.

Thus, for $b>b_{1}, f(k, 0)$ is a decreasing function of $k$. Since $b_{1}>b_{0}, f(2,0)<0$ for $u$ in the interval $r<u<a_{0}$. Therefore $f(k, 0)<0$ for $k \geqq 2$, (2) is negative for $r<u<a_{0}$, and thus $\theta(u)$ is not a totally regular mass function.

Proof of Theorem 3. Let $\mu(t)=\alpha_{1}(t, k+1, c) / \alpha_{1}(t, k, c)$. Then

$$
\mu(t)=\left(\frac{k}{k+1}\right)\left(1+\frac{c^{k}}{(t+c) \prod_{n=1}^{k-1}\left(t-c \alpha_{n}\right)}\right),
$$

where $\alpha_{n}=-1+\cos (2 n \pi / k)+i \sin (2 n \pi / k), n=1,2, \cdots, k-1$. Using a partial fraction decomposition of the second quantity in the parentheses, we may write it in the form

$$
\frac{c}{t+c}+\sum_{n=1}^{k-1} \frac{c \alpha_{n}}{k\left(t-c \alpha_{n}\right)}
$$

If $\theta(u)$ denotes the mass function of $\mu(t)$, then

For $0<u<1$,

$$
\theta(u)=\left\{\begin{array}{l}
\frac{1}{k+1}\left(k u^{c}-\sum_{n=1}^{k-1} u^{-c a_{n}}\right), 0 \leqq u<1 . \\
1, \quad u=1,
\end{array}\right.
$$

$$
\frac{d \theta}{d u}=\frac{c u^{c-1}}{k+1}\left(k+\sum_{n=1}^{k-1} \beta_{n}\right),
$$

where $\beta_{n}=\alpha_{n} u^{-c\left(\alpha_{n}+1\right)}$. Since $\beta_{n}$ and $\beta_{k-n}$ are complex conjugates,

$$
\sum_{n=1}^{k-1} \beta_{n}= \begin{cases}\sum_{n=1}^{(k / 2)-1}\left(\beta_{n}+\beta_{k-n}\right)-2 u^{c}, & k \text { even, } \\ \sum_{n=1}^{(k-1) / 2}\left(\beta_{n}+\beta_{k-n}\right), & k \text { odd }\end{cases}
$$




$$
\beta_{n}+\beta_{k-n}=-4 \sin \left(\gamma_{n}+(n \pi / k)\right) \sin (n \pi / k) u^{-c \cos (2 n \pi / k)},
$$

where $\gamma_{n}=-c(\log u) \sin (2 n \pi / k)$.

Since all values of $n$ for which $\cos (2 n \pi / k) \leqq 0$ give rise to terms bounded in $u$, consider only those terms of the series in (6) from 1 to $p$, where $p=[k / 4]$.

Let $s_{n}=\sin \left(\gamma_{n}+(n \pi / k)\right) \sin (n \pi / k)$. Then the series from 1 to $p$ may be written in the form

$$
\begin{aligned}
(-4) \sum_{n=1}^{p} s_{n} u^{-c \cos (2 n \pi / k)}=-4 u^{-c \cos (2 p \pi / k)}\left[s_{p}\right. \\
+u^{-c(\cos (2(p-1) \pi / k)-\cos (2 p \pi / k))}\left(s_{p-1}+\cdots\right. \\
+u^{-c(\cos (4 \pi / k)-\cos (6 \pi / k))}\left(s_{2}\right. \\
\left.\left.\left.+s_{1} u^{-c(\cos (2 \pi / k)-\cos (4 \pi / k))}\right) \cdots\right)\right]
\end{aligned}
$$

Pick a sequence of values $u_{m}$ of $u$ such that

$$
\begin{aligned}
\gamma_{1}+(\pi / k) & =(4 m-3) \pi / 2 ; \text { i.e., } \\
u_{m} & =\exp [\{(\pi / k)-((4 m-3) \pi / 2)\} / c \sin (2 \pi / k)] .
\end{aligned}
$$

Then, for each term of this sequence, $s_{1}=\sin (\pi / k)$. For each $k>4$, and all $m$ sufficiently large, each of the quantities in parentheses becomes unbounded.

Therefore $d \theta / d u<0$ for an infinite number of values of $a$ near 0 , and $\theta(u)$ is not a totally regular mass function.

The restriction $k>4$ is a natural one, in order to guarantee that there will be at least one value of $n$ for which $\cos (2 n \pi / k)>0$.

Moreover, formulas (5) and (6) enable one to give a shorter proof of Theorem 4.8 of [3], which I shall now do.

For $k=3, \theta(u)$ is a totally regular mass function, provided we can show that (5) is nonnegative. Using (6) this is equivalent to the condition that $f(u) \geqq 0$, where

$$
\begin{aligned}
f(u) & =3-4 u^{-c \cos (2 \pi / 3)} \sin \left(\gamma_{1}+(\pi / 3)\right) \sin (\pi / 3) \\
& =3-2 \sqrt{ } 3 u^{c / 2} \sin \left(\gamma_{1}+(\pi / 3)\right) .
\end{aligned}
$$

For $0<u<1, f(u)$ possesses a minimum at each value of $u$ for which $\gamma_{1}=2 m \pi$; i.e., $\quad u=\exp (-4 m \pi / \sqrt{ } 3 c) f(u=\exp (-4 m \pi / c \sqrt{ } 3))=3(1-\exp (-2 m \pi / \sqrt{ } 3))$ $>0$. Since $f(0)=1$, the oscillations of $f$ near 0 remain positive. If $u$ lies in the interval $0 \leqq \gamma_{1}+(\pi / 3) \leqq \pi$, then $\exp (-4 \pi / 3 c \sqrt{ } 3) \leqq u \leqq 1$, and $f^{\prime}(u)<0$ for all $u$ in this interval. Since $f(1)=0, f(u)$ is nonnegative in this interval. We have now shown that $f(u) \geqq 0$ for all $u$ in the interval $[0,1]$, and hence $\theta(u)$ is a totally regular mass function.

For $k=4$, let

$$
f(u)=4-2 \sqrt{ } 2 \sin \left(\gamma_{1}+(\pi / 4)\right)-2 u^{c}
$$


Let $u_{m}$ denote those values of $u$ for which

$$
\gamma_{1}+(\pi / 4)=(2 m-1) \pi / 2, m=1,2,3, \cdots ; \text { i.e., } u_{m} \exp (-(4 m-3) \pi / 4 c) .
$$

Partition $0<u<1$ by means of $0<\cdots<u_{2 m+2}<u_{2 m+1}<u_{2 m}<u_{2 m-1}<\cdots<1$. We note that $\sin \left(\gamma_{1}+(\pi / 4)\right)$ has a maximum value at $u_{2 m-1}$ and a minimum value at $u_{2 m}$, and $u^{c}$ is monotone increasing for $0<u<1$. Hence, for $u_{2 m+1} \leqq u$ $\leqq u_{2 m}, f(u) \geqq 4-2[\sqrt{ } 2+\exp (-8 m-3) \pi / 4]>0$. For $u_{2 m} \leqq u \leqq u_{2 m-1}$, $f(u) \geqq 4-2[\sqrt{ } 2+\exp ((-8 m-7) \pi / 4)]>0$. Since $f(0)=4>0$, all oscillations near 0 remain positive. For $\exp (-\pi / c) \leqq u \leqq 1, f(u) \geqq 4-2(\sqrt{ } 2(1 / \sqrt{ } 2)+1)$ $=0$. Therefore $\theta(u)$ is a totally regular mass function.

Clearly $1 / \mu(t)$ cannot be totally monotone, since $\lim _{t \rightarrow \infty} 1 / \mu(t)=(k+1) / k>1$. I wish to thank Robert N. Buchal, Argonne National Laboratory, for his assistance with the proofs of Theorems 1 and 2 .

I also wish to thank Argonne National Laboratory for permitting me to use their IBM 704 computer to obtain preliminary results connected with the proofs of these theorems.

\section{REFERENCES}

1. H. L. Garabedian, E. Hille and H. S. Wall, Formulation of the Hausdorff inclusion problem, Duke Math. J. 8 (1941), 193-213.

2. H. J. Greenberg and H. S. Wall, Hausdorff means included between $(C, 0)$ and $(C, 1)$, Bull. Amer. Math. Soc. 48 (1942), 774-783.

3. B. E. Rhoades, Hausdorff summability methods, Trans. Amer. Math. Soc. 101 (1961) $396-425$.

ARgonne National Laboratory,

ARGONNE, ILLINOIS

LAFAyetTe College,

Easton, Pennsylvania 